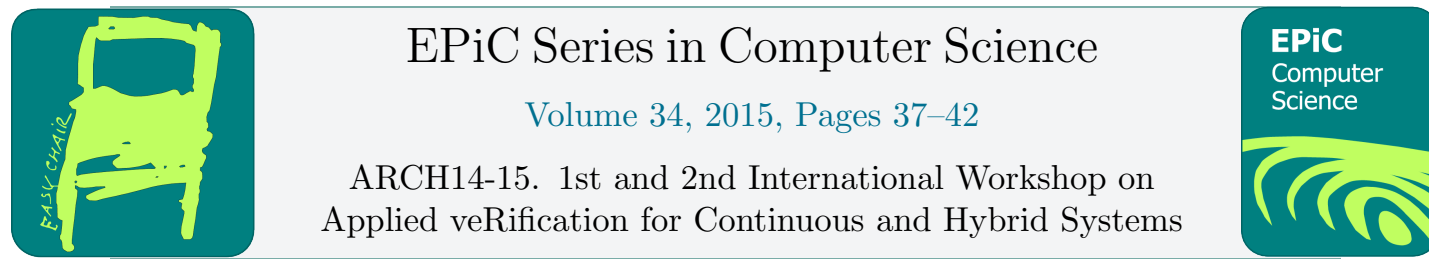

\title{
Networked Cooperative Platoon of Vehicles for Testing Methods and Verification Tools
}

\author{
Ibtissem Ben Makhlouf and Stefan Kowalewski \\ Informatik 11 - Embedded Software RWTH Aachen University \\ Ahornstrasse 55, 52074 Aachen, Germany \\ makhlouf, kowalewski@embedded.rwth-aachen.de
}

\begin{abstract}
A practical benchmark for testing analysis and verification methods for continuous as well as hybrid systems is suggested. It consists of a platoon of controlled vehicles. The vehicles exchange information via a communication network that is subject to failure of single nodes or a complete loss of communication. The worst case scenario corresponding to the transition from a fully functioning communication between the vehicles to a total loss of communication is considered in particular. The safety properties of the considered networked platoon are investigated. The system is modeled as a hybrid automaton. The continuous part represents the dynamical behavior of the platoon and the discrete part are spontaneous events related to the switching communication topology. The proposed example is a linear time invariant system.

Category: academic

Difficulty: challenge
\end{abstract}

\section{Context and Origins}

The benchmark suggested here has been already proposed as a practical example for testing methods for computing reachable sets for continuous as well as for hybrid systems. In [2], a performance comparison between approximation methods based on support functions and zonotopes with invariant computation methods combining linear matrix inequality (LMI) technique and ellipsoids was presented. The tightness of the approximation and the computation effort were particularly taken into consideration. With the platoon example, the goal was to test which of these methods provides the shortest safe gaps between the vehicles. This example is therefore challenging because of its state dimension.

\section{Benchmark Description and Testing Goals}

We consider now the platoon of $n$ vehicles illustrated in Figure 1. The spacing error $e_{i}$ is in this context defined as the difference between the distance $d_{i}$ of the truck $i$ to its predecessor and a reference distance $d_{r e f, i}$. The principal goal of the reachability analysis for this system is to determine a lower bound for $d_{r e f, i}$ assuring collision-free driving. We assume that each 
vehicle $i$ is equipped with on-board sensors to capture the own relative distance to the vehicle ahead, the relative velocity $\dot{e}_{i}$ and the acceleration $a_{i}$. Moreover, the collected data from other platoon members can be also accessed via WLAN communication. The controller design aims

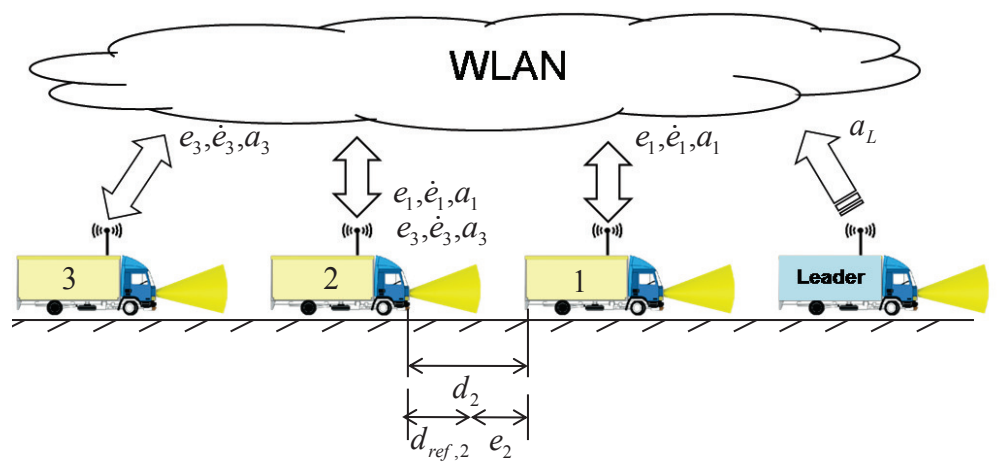

Figure 1: WLAN cooperative platoon with three vehicles and a leader ahead.

at stabilizing the system while assuring a good disturbance rejection in terms of small and safe spacing errors $e_{i}$ with a reasonable control effort. The platoon is modeled as concentrated masses and the effective acceleration of each vehicle within the platoon is governed by the drivetrain dynamics approximated by a linear first order filter $[5,4]$ and is described as follows

$$
\begin{aligned}
\ddot{e}_{i} & =a_{i-1}-a_{i}, \\
\dot{a}_{i} & =-1 / T_{i} \cdot a_{i}+1 / T_{i} \cdot u_{i},
\end{aligned}
$$

where $T_{i}$ is the time constant of the drivetrain of vehicle $i$ and $u_{i}$ the corresponding control signal. To express the control objectives, we consider different transfer functions and mappings as defined in $[5,4]$. With the definition of the following transfer function, for example,

$$
G_{i}(s)=\frac{e_{i}(s)}{e_{i-1}(s)},
$$

the string stability ensuring $e_{i} \leq e_{j}$ for $i \geq j$ can be guaranteed under the condition $\left\|G_{i}\right\|_{\infty}<1$. On the other side, to minimize the upper bounds of the overshoots on velocity and acceleration with respect to the acceleration of the leader $a_{L}$ (considered here as a disturbance) the following transfer functions are defined

$$
F_{v, i}(s)=\frac{\left(v_{i}-v_{L}\right)(s)}{a_{L}(s)}, F_{a, i}(s)=\frac{a_{i}(s)}{a_{L}(s)} .
$$

The conditions $\left\|F_{v, i}(s)\right\| \leq \gamma_{v}$ and $\left\|F_{v, i}(s)\right\| \leq \gamma_{a}$ maintain the velocities and accelerations for all the followers respectively below $\gamma_{v}\left\|a_{L}\right\|_{\infty}$ and $\gamma_{a}\left\|a_{L}\right\|_{\infty}$. The control problem may be then formulated as a mixed $H_{2} / H_{\infty}$ problem (cf.[5]):

$$
\begin{aligned}
& \min \alpha .\|F\|_{\infty}+\beta .\|H\|_{2} \text { s.t. } \\
& \left\|G_{i}\right\|_{\infty}<1 \forall i \\
& \left\|F_{., i}\right\|_{\infty}<\gamma . \forall i .
\end{aligned}
$$

where $F$ is either $F_{v, i}(s), F_{a, i}(s)$ or a combination of both and $H: a_{L} \mapsto\left(e_{1}, \ldots, e_{n}, u_{1}, \ldots, u_{n}\right)^{T}$ the mapping of $a_{L}$ to errors and control effort. The $\|\cdot\|_{2}$-norm minimization of $H$ guarantees 
hence for short distance errors with a low control effort. The minimization problem (5) results in a feedback matrix $K$ verifying $u=K x$, where $x=\left[\cdots e_{i}, \dot{e}_{i}, a_{i} \cdots\right]^{T} \in \mathbb{R}^{3 n}$ is the state vector and $u$ is the control vector of the whole platoon. The closed loop system can therefore be described by the following differential equation

$$
\dot{x}=A x+B a_{L},
$$

where $A$ is a constant system matrix, $B$ is a constant input matrix and $a_{L}$ the acceleration of the leader considered here as an uncertain input $[3,1]$.

We consider as case study a three vehicle platoon with a manually driven leader ahead described by (6). The time constant of the drivetrain is assumed for the following computed matrices to be equal to $T_{i}=0.5 \mathrm{~s}$ for all platoon members. The state vector is then $x=\left[e_{1}, \dot{e_{1}}, a_{1}, e_{2}, \dot{e_{2}}, a_{2}, e_{3}, \dot{e_{3}}, a_{3}\right]$ and the acceleration of the leader $a_{L} \in I$ with $I \subseteq[-9,1] \mathrm{ms}^{-2}, a_{L}^{b r a k e}=-9 \mathrm{~ms}^{-2}$ is the maximum truck deceleration and $a_{L}^{a c c}=1 \mathrm{~ms}^{-2}$ is the maximum truck acceleration.

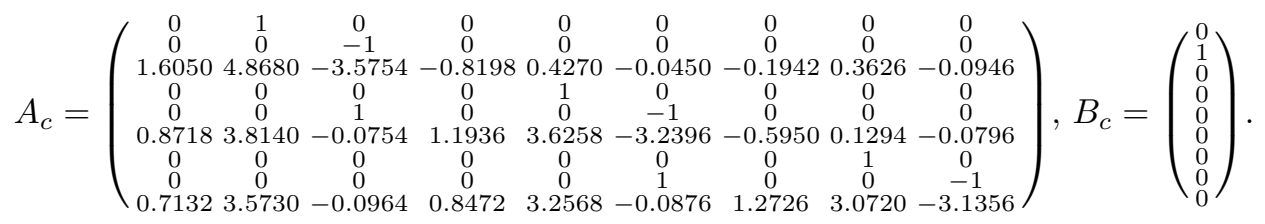

The initial set is assumed to be close to the origin.

The goal is to determine the minimum allowable safe gaps inside the platoon.

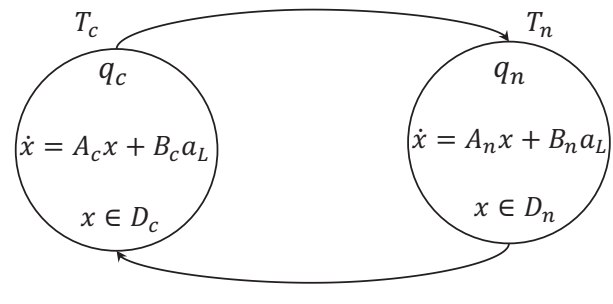

(a)

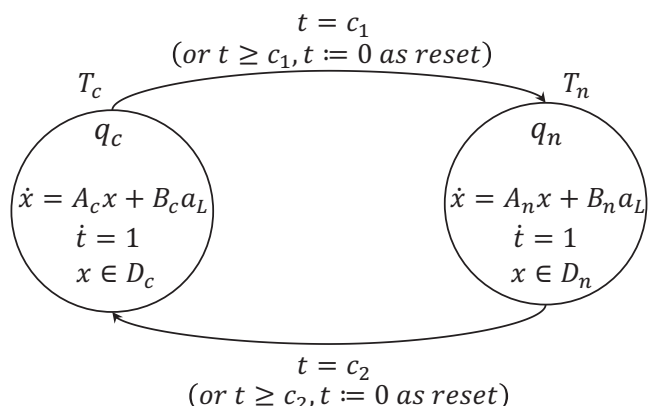

(b)

Figure 2: Hybrid models for the worst case scenario. (a) Guard-free transitions. (b) Time trigged transitions.

Of particular interest are these values when the communication between the vehicles suddenly fails while driving. In fact, the interconnection topology within the platoon is modeled with a directed graph $G=(V, E)$, defined by vertices $V$ and edges $E$. The $i^{t h}$ vertex represents the $i^{\text {th }}$ vehicle and the edge $(i, j)$ indicates that vehicle $j$ receives information from vehicle $i$. This graph is described by the adjacency matrix $R=\left[r_{i j}\right]$ [4]. In this context, $R$ is called the communication matrix of the platoon and is defined as follows:

$$
r_{i j}:= \begin{cases}1, & \text { if } \mathrm{j} \text { receives information from i } \\ 0, & \text { if not }\end{cases}
$$

The on-board sensors inform each vehicle about its actual state. If we assume these sensors to be reliable, the diagonal of the communication matrix is therefore everywhere equal to one. To 


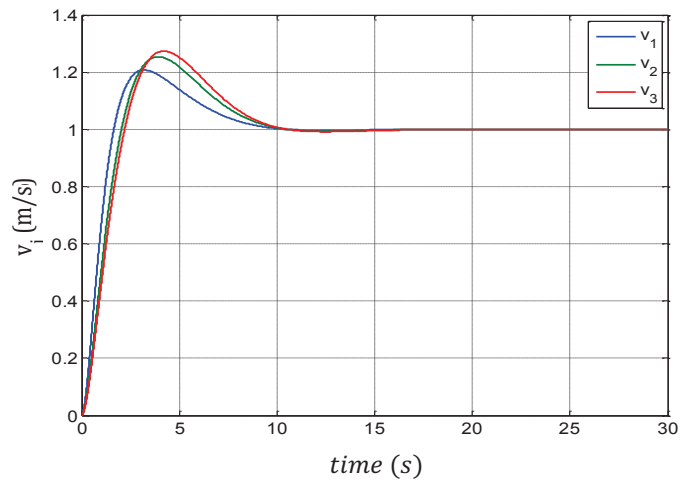

(a)

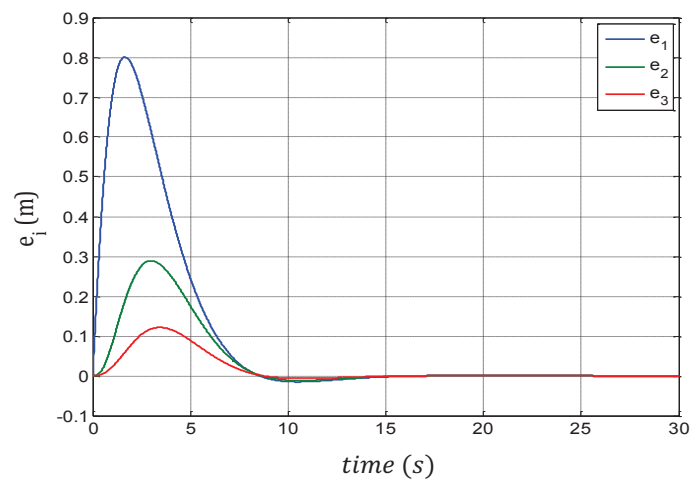

(b)

Figure 3: Platoon response to a velocity step of $1 \mathrm{~m} / \mathrm{s}$ using just $H_{2}$ optimization.

take into account the communication failures in the controller design, the loss of information is expressed by forcing zeros in appropriate coefficients of the feedback matrix $K$. In case of total dropout of the communication the closed loop system describing the controlled platoon is given by

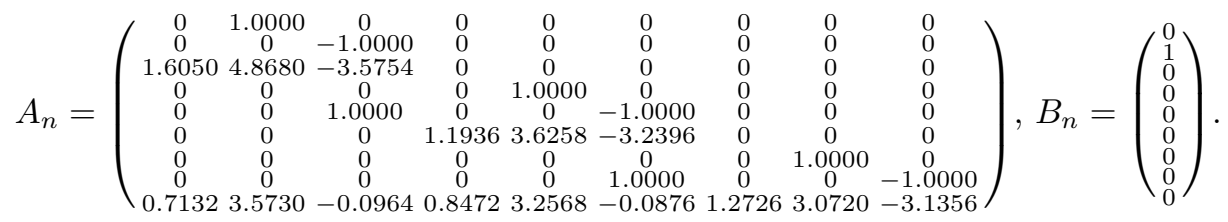

This behavior can be modeled as a hybrid automaton. The dynamics of the closed loop system constitute thereby the continuous states. The communication breakdowns, however, trigger the discrete switches from one continuous state to another. We consider the following hybrid automata describing the worst case scenario of a hazard loss of communication between all members of the platoon. Discrete switches can be modeled as spontaneous, true or guard free transitions (Figure 2 (a)) [2]. Otherwise, a clock can be added making therefore the switching dependent on time (Figure $2(\mathrm{~b})$ ). Alternatively, switching can be forced if the algorithm computing the reachable set attains a fixpoint. That corresponds, in this context, to an invariant reachable set. Simulations during the control design, like those of Figure 3, help to determine the time horizon $T$ after which the controlled system reaches a stable region. For our case study, we have $T=12 \mathrm{~s}$. In this example, the sets $D_{c}$ and $D_{n}$ corresponding respectively to the state space domains of the continuous states $q_{c}$ and $q_{n}$ are taken, for this particular application, equal to $\mathbb{R}^{9}$. Otherwise the user is free to make a different but suitable choice. Furthermore, the decision about the values of the time constants $c_{1}$ and $c_{2}$ of the hybrid model in Figure 2 (b) are also left to the user.

\section{Key Observations}

For a platoon of three autonomous vehicles with a manually driven leader ahead, the state vector of the system is $x=\left[e_{1}, \dot{e_{1}}, a_{1}, e_{2}, \dot{e_{2}}, a_{2}, e_{3}, \dot{e_{3}}, a_{3}\right]$. The computation can start with the origin $0_{\mathbb{R}^{9}}$ or with a neighborhood of the origin. The maximum allowed uncertain input set is $[-9,1] \mathrm{ms}^{-2}$. 


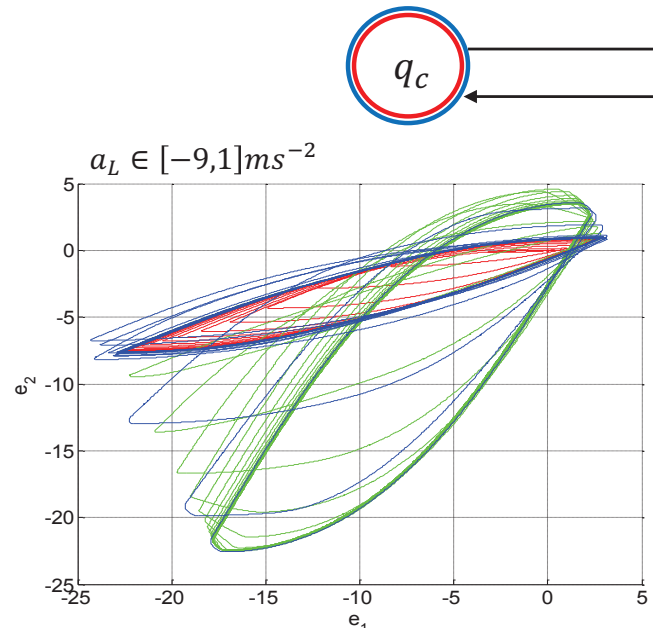

(b)

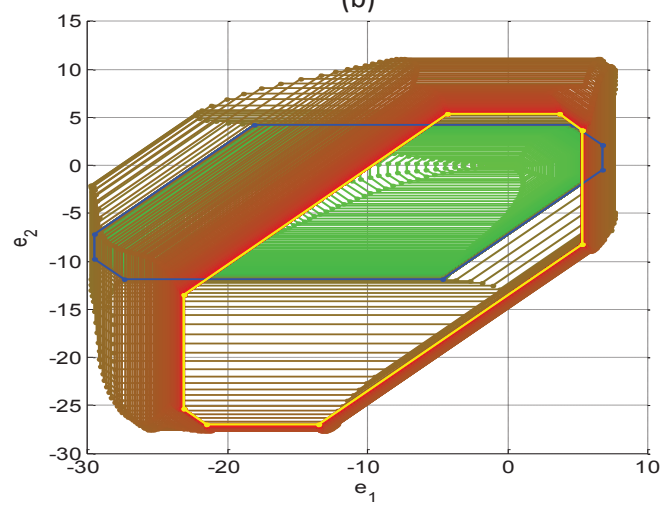

(d)

\section{$q_{n}$}

(a)

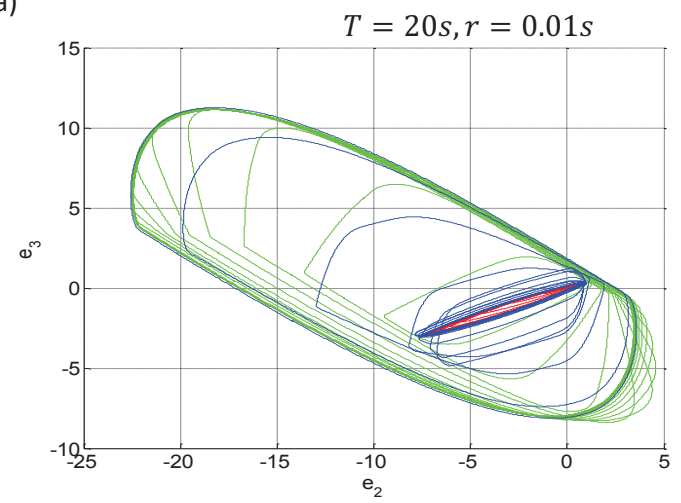

(c)

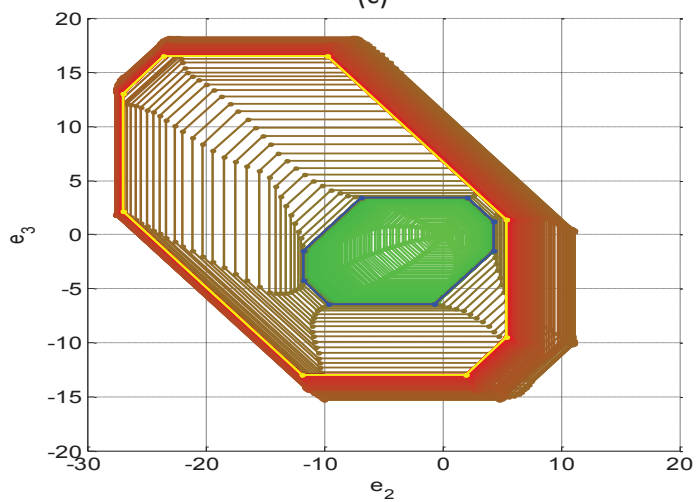

(e)

Figure 4: Different projections of the reachable set computed using a time step $r=0.01 \mathrm{~s}$, a time horizon $T=20 s$ and an uncertain input $a_{L} \in[-9,1] \mathrm{ms}^{-2}$. (a) Hybrid automaton of the considered scenario. (b)-(c) Projections of the reachable set approximated by zonotopes. (d)-(e) Projections of the reachable set obtained by using support functions

- We are concerned with finding the shortest gaps $e_{1 m i n}, e_{2 m i n}, e_{3 m i n}$ within the platoon which correspond to the lower bounds of the reachable set in the corresponding directions of the canonical basis. Therefore to avoid collisions inside the platoon, the following condition must be fulfilled

$$
e_{1}>e_{1 \min } \wedge e_{2}>e_{2 \min } \wedge e_{3}>e_{3 \min }
$$

- We experimented with our implementation based on zonotopes and the recent one using support functions. The results are illustrated in Figure 4. We note that for the method based of zonotopes the values $e_{1 \min }=25 \mathrm{~m}, e_{2 \min }=25 \mathrm{~m}$ and $e_{3 \min }=10 \mathrm{~m}$ can be taken as safe gaps. For the support function large safe gaps must be considered because of the results of Figure $4(\mathrm{~d})-(\mathrm{e})$. In this case, we have $e_{1 \min }=30 \mathrm{~m}, e_{2 \min }=30 \mathrm{~m}$ and $e_{3 \min }=16 \mathrm{~m}$. 
- For verification purposes, we propose, for example, the unsafe condition

$$
e_{1}<-e_{1 \min } \vee e_{2}<-e_{2 \min } \vee e_{3}<-e_{3 \min }
$$

\section{Outlook}

We consider in this paper a practical benchmark. The issues under focus include the tightness of the obtained reachable set, the efficiency and complexity of the implemented methods. We aim in a future work to use the same formulation for the control design to propose a platoon benchmark with a scalable number of vehicles in order to test the limits of applicability of tools. We will stepwise increase the dimension of the benchmark, measure the time and the space complexity and determine the maximum system dimension feasible for each tool.

\section{Acknowledgment}

Special thanks go to Jan P. Maschuw (Institute of Automatic Control, RWTH Aachen University, Germany), the control designer of the platoon, for the fruitful discussions and the constructive cooperation.

\section{References}

[1] I. Ben Makhlouf, H. Diab, and S. Kowalewski. Safety verification of a controlled cooperative platoon under loss of communication using zonotopes. In ADHS 2012, Eindhoven, NL, pages 333338. Inproceeding of the 4th IFAC Conference on Analysis and Design of Hybrid Systems (ADHS 12), 2012.

[2] I. Ben Makhlouf, P. Hänsch, and S. Kowalewski. Comparison of reachability methods for uncertain linear time-invariant systems. In ECC13: European Control Conference, Zurich, Switzerland July 17-19,, pages 1101-1106, 2013.

[3] I Ben Makhlouf, J. P. Maschuw, P. Hänsch, H. Diab, S. Kowalewski, and D. Abel. Safety verification of a cooperative vehicle platoon with uncertain inputs using zonotopes. In 18th IFAC World Congress, 2011, Milano, Italy, pages 9769-9774, 2011.

[4] J. P. Maschuw and D. Abel. Longitudinal vehicle guidance in networks with changing communication topology. In AAC 2010, IFAC-Symposium Advances in Automotive Control, München, Jul. 2010.

[5] J. P. Maschuw, G. C. Keßler, and D. Abel. LMI-based control of vehicle platoons for robust longitudinal guidance. In IFAC World Congress 2008, Seoul, Jul. 2008. 